\title{
An automatic procedure for extracting demisyllables from isolated monosyllabic source words for use in speech synthesis-by-rule of Thai
}

\author{
Chatchavalit Saravari and Satoshi Imai \\ Research Laboratory of Precision Machinery and \\ Electronics, Tokyo Institute of Technology, \\ Nagatsuta, Midori-ku, Yokohama, 227 Japan
}

(Received 4 July 1983)

\begin{abstract}
In this paper, we describe a procedure for automatically extracting demisyllables (DSs) from Thai isolated source words of the forms consonant-vowel, vowel-consonant, and vowel. Routines that are particular to each category of source words are used to establish DS boundary locations. All segmental cues are derivable from the cepstrum representing a log spectral envelope that approximately passes through peaks of the fine structure, which have been manually determined based on spoken source words of one male talker. The automatic procedure has been used to extract DSs from spoken source words of two new talkers: one male and one female. Manual comparisons have shown that automatically obtained DS boundary locations were within three frames ( 1 frame is $10 \mathrm{~ms}$ ) from the corresponding locations obtained manually by a trained native linguist. For the male talker, $94.2 \%$ of the automatically obtained locations coincided with the corresponding manually obtained locations, and $3.5 \%, 1.9 \%$, and $0.4 \%$ differed by one, two, and three frames, respectively; for the female talker, $95.7 \%$ coincided, and $3.3 \%, 0.8 \%$, and $0.2 \%$ differed by one, two, and three frames, respectively. The above results are comparable with the results of using the automatic procedure to extract the DSs from the source words of the old talker: $97.5 \%$ coincided, and $2.3 \%$ and $0.2 \%$ differed by one and two frames, respectively. These results indicate that the automatic procedure promises a very good first estimate set of DSs.
\end{abstract}

PACS number: 43. 70. Jt

\section{INTRODUCTION}

Our recent studies ${ }^{1-3)}$ have shown that speech synthesis on a basis of concatenating demisyllable cepstral parameters and transmitting the concatenated data to the log magnitude approximation (LMA) digital filter as the filter coefficients promises a favorable outcome in producing Japanese and Thai messages. In such courses, a system is designed to convert from a sequence of input symbols to a continuous speech waveform: First, a preprocessor in the system chooses the demisyllable cepstral parameters to be concatenated from an inventory. Then, based on stored synthesis rules, a synthesis program determines the transformation from the sequence of input symbols to a continuous set of control signals for driving a speech synthesizer. Finally, the speech synthesizer converts from the continuous control signals to the continuous speech waveform. In the speech synthesizer, a time varying digital filter (the LMA filter) with an impulse train with a spacing equal to a given pitch period as its input for a voiced speech portion or a train of equally spaced impulse with random polarity as its input for an unvoiced speech portion is used as the synthesis filter. The switching from one impulse train to the other is controlled by a voiced-unvoiced sign. The LMA filter is characterized by the con- 
catenated cepstral parameters. The continuous speech waveform is the output of the LMA filter.

One of the factors that determine the quality of synthetic speech is the process of extracting demisyllables (DSs) from their source words; the DSs must be extracted by means of proper rules for defining them and an accurate segmentation procedure.

As yet, the process of extracting DSs from their source words for use in a speech synthesis system is manual; but at that, extracting DSs manually by a trained linguist (looking at waveforms, spectra, etc.) appears to be most accurate. ${ }^{1,2)}$ However the manual process is generally wearisome and timeconsuming; it will be unwieldy for an amateur to use such process without training. In addition, when computer voice for many talkers is required, creating a suitable set of DSs for each talker manually seems to be a tedious task. Accordingly, alternative approaches to creating the DS inventory should be thought about.

One of the approaches is to use an automatic segmentation procedure to estimate the DS boundary locations first, and then adjust, if necessary, the automatically obtained DS boundary locations manually until all criteria for defining the DSs are satisfied. An accurate automatic segmentation can much reduce or even eliminate the manual work. With the aid of an automatic procedure, it can be thought that even an amateur can easily create a suitable set of DSs for use in a speech synthesis system. We believe that automatic extracting speech units from their source words will become a challenging subject in courses of speech synthesis-by-rule and recognition in years to come.

Rabiner et al. $^{4}$ ) have recently proposed a procedure for using DS reference patterns, extracted from spoken utterances for one talker and automatically creating DS reference patterns for a new talker. The obtained DS reference patterns are to be used in a recognition system. The procedure is based on dynamic time warping alignment of the spoken utterances (each containing the relevant DS), and the assumption that the optimum warping path identifies the best matching DS within the utterance. They found that recognition accuracies from reference patterns based on automatically extracted DSs were not so much $(2 \% \sim 5 \%)$ worse than accuracies from reference pattern based on hand correction applied to the DSs. They remarked that this is because small boundary errors do not affect the re- cognition accuracy, but that for speech synthesis systems the boundary errors would be more harmful and would have to be detected and corrected for best results.

The present study and the study by Rabiner et al. are mostly common in idea but different in designing the automatic segmentation procedure and in applying the DS units. In the present procedure, the DS boundary locations are automatically established by means of segmental cues derivable from the cepstrum representing a log spectral envelope that approximately passes through peaks of the fine structure. ${ }^{5)}$ Here, the extracted DSs are to be used as basic units in a speech synthesis system.

Different from segmenting unknown speech into demisyllables of a recognition system, the present segmentation procedure, as well as Rabiner's extracts demisyllables from known source words. The present procedure uses cues and routine particular to each source word, which have been chosen based on visual displays of spoken source words of one male talker. The cues and routine are those expected to be able to be applied to any talker. In our previous short letter, ${ }^{6)}$ we have introduced some segmental cues that may be used in an automatic segmentation procedure. The present paper is a continuation of that letter, which describes in details: the rules for defining the DSs to be used, the segmental cues, the procedure for automatically extracting DSs from source words, and the performance of the automatic procedure.

Figure 1 shows a block diagram of the procedure. The cepstra of isolated source words are computed and stored. A symbolic input of the source word from which a DS is to be extracted calls the analyzed

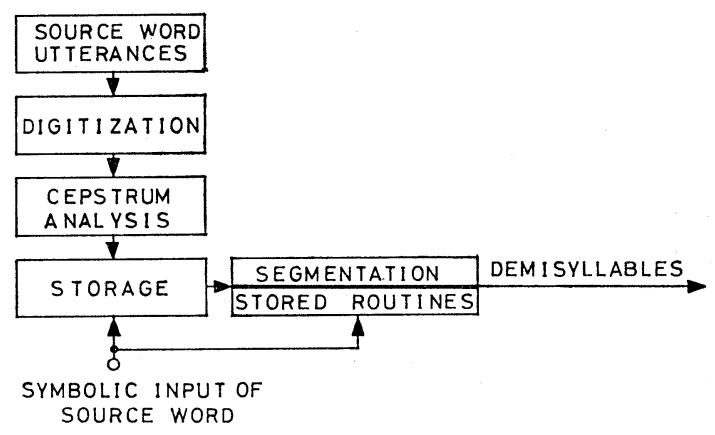

Fig. 1 Block diagram of automatic procedure for extracting demisyllables from isolated monosyllabic source words. 


\section{SARAVARI and S. IMAI: AUTOMATIC EXTRACTING DEMISYLLABLES}

cepstra of the word from the storage, and simultaneously chooses a proper segmentation routine to be used. The routine then establishes the DS boundary locations within the word.

\section{SOURCE WORDS AND ANALYSIS METHOD}

The list of source words contains words of the forms consonant-vowel (CV), vowel-consonant (VC), and vowel (V). Each source word is to be uttered in isolation in the level tone, which has rather flat fundamental frequency movement. For the $\mathrm{CV}$ form, the initial $\mathrm{C}$ can be any of these following phonemes: the voiced stops $/ \mathrm{g} /, / \mathrm{b} /$, and $/ \mathrm{d} /$; the unvoiced stops $/ \mathrm{k} /, / \mathrm{t} /, \mid \mathrm{t} /, / \mathrm{p} /$, and $/ \mathrm{p} /$; the nasals $/ \mathrm{m} /, / \mathrm{n} /$, and $/ \mathrm{g} /$; the voiced affricate $/ \mathrm{dzh} /$ (since this sound can be modeled as the concatenation of the stop /d/ and the fricative / $\mathrm{zh} /$, we shall denote it as shown); the unvoiced affricate $/ \mathrm{t} / /$; the unvoiced fricatives $/ \mathrm{f} /, / \mathrm{s} /$, and $/ \mathrm{h} /$; the semivowels $/ 1 /, / \mathrm{w} /$, and $/ \mathrm{y} /$; and the trill $/ \mathrm{r} /$ (the consonant sound pronounced with a quick vibration of tongue); the final $\mathrm{V}$ can be any of these following vowels: $/ \mathrm{I} /$, $/ \mathrm{e} /, / æ /, / \mathrm{a} /, / 3 /, / \mathrm{l} /, / \mathrm{u} /, / \mathrm{U} /$, and $/ \mathrm{o} /$. For the $\mathrm{VC}$ form, the initial $\mathrm{V}$ can be any of the vowels shown above; the final $\mathrm{C}$ can be any of these following consonants: the voiced stops $/ \mathrm{g} /, / \mathrm{d} /$, and $/ \mathrm{b} /$; and the nasals, $/ \mathrm{m} /, / \mathrm{n} /$, and $/ \mathrm{n} /$. (The contrast between the stops $/ \mathfrak{i} /$ and $/ \mathrm{t} /$, as well as between $/ \mathrm{p} /$ and $/ \mathrm{p} /$, is that for the former, more pressure is built up behind the occlusion and the air is released with much shorter period of aspiration. Since we could not find proper corresponding symbols in the International Phonetic Alphabet for $/ \mathrm{t} /$ and $/ \mathrm{p} /$, we shall denote them as shown).

In the analysis, the input speech is sampled with a $10 \mathrm{kHz}$ sampling rate and digitized by a 12-bit quantizer. In the digitization, the gain of the input speech is adjusted so that full quantization range was used without clipping. At least $150 \mathrm{~ms}$ of the interval of silence in front of each source word is to be included. The cepstrum analysis is performed every $10 \mathrm{~ms}$, in which a 25.6-ms Hamming window is used. Each analysis frame of speech is represented by 26 cepstral values (the cepstral gain and the first 25 coefficients) that are adapted to represent a log spectral envelope that approximately passes through peaks of the fine structure. ${ }^{5)}$

The rules for defining the demisyllables for use in a speech synthesis system are as follows.
1) A CV demisyllable whose initial consonant is voiced and rather continuant (a nasal or a semivowel) comprises the transition from the beginning of the most steady state portion of the consonant to the point $80 \mathrm{~ms}$ behind the vowel onset.

2) $\mathrm{A} \mathrm{CV}$ demisyllable whose initial consonant is unvoiced and continuant (a fricative) comprises the transition from $100 \mathrm{~ms}$ in front of the point of the most rapid transition from the consonant to the vowel to the point $80 \mathrm{~ms}$ behind the vowel onset.

3) A CV demisyllable whose initial consonant is a dynamic sound (a stop, an affricate, or the trill) comprises the transition from the onset of the consonant to the point $80 \mathrm{~ms}$ behind the vowel onset.

4) A VC demisyllable whose final consonant is a nasal comprises the transition from the point $60 \mathrm{~ms}$ in front of the vowel offset (the vowel end) to the end of the most steady state portion of the consonant.

5) A VC demisyllable whose final consonant is a stop comprises the transition from the point 60 $\mathrm{ms}$ in front of the vowel offset to the end of the final consonant.

6) A V demisyllable comprises the first $50-\mathrm{ms}$ segment of the most steady state portion of a $\mathrm{V}$ source word. This segment may be too short to be called demisyllable. But since a meaningful monosyllabic word can be produced from such unit, we shall define it as a demisyllable.

\section{SEGMENTAL CUES}

The cues used in the segmentation procedure are derivable from the cepstral values. The cepstrum of frame $\nu, v_{\nu}(m)$, is defined as the Fourier cosine coefficients of the log spectral envelope, $V_{2}(k)$, as follows:

$$
v_{2}(m)=\frac{1}{N} \sum_{k=0}^{N-1} V_{2}(k) \cos \left(\frac{2 \pi}{N} m k\right), \quad 0 \leq m \leq M .
$$

Based on visual displays of several parameters derivable from the cepstra of the source words spoken by a male talker (CS), the following parameters are seen to have good performance in segmentation (some cues that are prominent for some instance may be less prominent than other cues for other instance; in the segmentation procedure, we have tried to use prominent cue(s) that is (are) particular to each instance). 


\subsection{Cepstral Gain}

Taking $m=0$ in Eq. (1) we have

$$
v_{\nu}(0)=\frac{1}{N} \sum_{k=0}^{N-1} V_{\nu}(k) .
$$

This term is the cepstral gain of frame $v$, which is the average of all the log spectral envelope components. We use $v_{2}(0)$ as a cue signaling the power of frame $\nu$.

\subsection{First Cepstral Coefficient}

The cepstral coefficients, $v_{2}(m), 1 \leq m \leq M$; are the averages of cosine-weighted log spectral envelope components. Figure 2 shows the functions

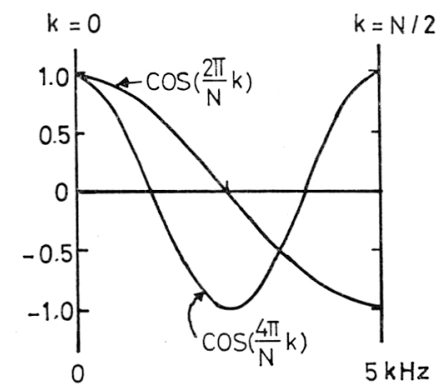

Fig. 2 Values that weight $\log$ spectral envelope components in the region from $k=0$ to $k=N / 2$ for obtaining the first $\left[\right.$ the function is $\left.\cos \left(\frac{2 \pi}{N} k\right)\right]$ and the second

$$
\left[\text { the function is } \cos \left(\frac{4 \pi}{N} k\right)\right]
$$
cepstral coefficients. that weight the $\log$ spectral envelope components within the range from $k=0$ to $k=N / 2$ (here $0 \sim 5$ $\mathrm{kHz}$ ) for obtaining

$$
\begin{aligned}
& v_{2}(1)\left[\text { the function is } \cos \left(\frac{2 \pi}{N} k\right)\right] \text { and } \\
& v_{2}(2)\left[\text { the function is } \cos \left(\frac{4 \pi}{N} k\right)\right] .
\end{aligned}
$$

A cepstral coefficient can be considered as representing the intensity of log spectral envelope components seen through a window.

Due to the effect of $\cos \left(\frac{2 \pi}{N} k\right)$ weighting, we found that $v_{2}(1)$ can be used as a cue signaling the transition from a fricative to the vowel that follows it. Figure 3 shows the waveforms, and time variations of $v_{2}(0)$ and $v_{2}(1)$ of the spoken utterances of /su/ of three talkers: two males, CS and PH [Figs. 3 (a) and 3 (b), respectively], and a female, OS [Fig. 3 (c)]. For every talker, the initial /s/ shows relatively small $v_{2}(1)$ values; note that the values of $v_{\nu}(1)$ during $/ \mathrm{s} /$ are even smaller than those during the interval of silence. During the transitional region, $v_{2}(1)$ rapidly rises toward the value at the vowel onset, which is considerably larger than the values during $/ \mathrm{s} /$. The variation of $v_{2}(1)$ during the transitional region appears to be more prominent than are the variation of $v_{2}(0)$ (the cepstral gain), especially for the two male talkers.

\subsection{Second Cepstral Coefficient}

Due to the effect of $\cos \left(\frac{4 \pi}{N} k\right)$ weighting, we found that $v_{2}(2)$ can be used as a cue signaling the
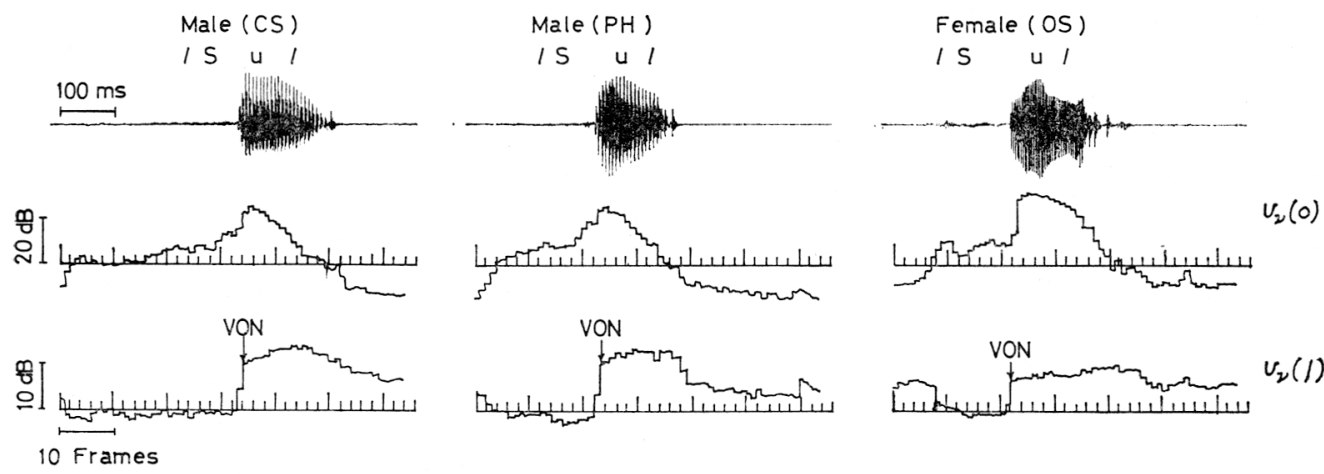

(a)

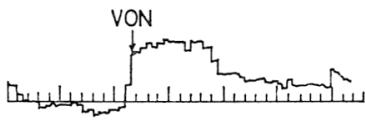

(b)

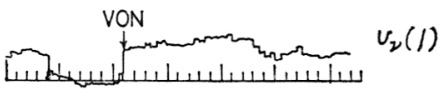

(c)

Fig. 3 Waveforms together with time variations of $v_{2}(0)$ and $v_{2}(1)$ of utterances of /su/ spoken by three talkers: two males, CS (a) and PH (b), and one female, OS (c). 


\section{SARAVARI and S. IMAI: AUTOMATIC EXTRACTING DEMISYLLABLES}

transition from an initial semivowel to the vowel that follows it, which is typically difficult to determine, even manually. Figure 4 (a) shows the waveforms, and time variations of the values of $v_{\nu}(0)$, $v_{2}(1)$, and $v_{2}(2)$ of the utterances of /yu/ of the three talkers whereas Fig. 4 (b) shows those of the utterances of $/ \mathrm{yI} /$. The frame marked VON within each word is the vowel onset established based on visual displays of the speech waveform and log spectral envelopes by a trained linguist. It is seen that, regardless of talker, $v_{2}(2)$ is minimum at the onset of both the following / $/$ /, which has the highest rising rate of $v_{2}(2)$, and the following $/ \mathrm{I} /$, which has the smallest rising rate of $v_{2}(2)$. Within each of the utterances, the transition from the semivowel to the vowel that follows it can be clearly seen through $v_{2}(2)$ as an rapid downward change; even the transition of $/ y /$ to $/ I /$, which is quite smooth, can be seen.
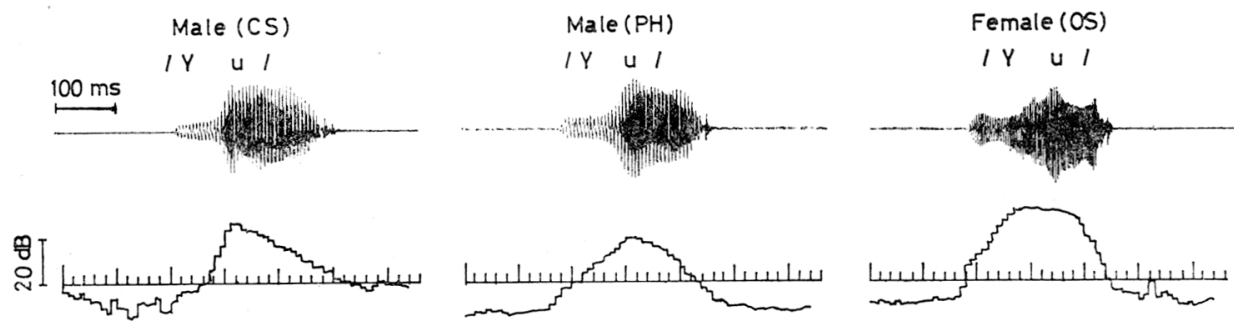

$v_{2}(0)$
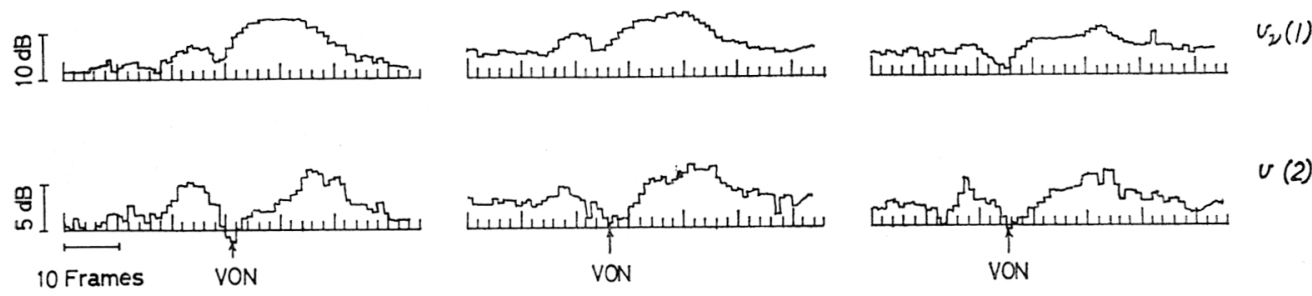

$v(2)$

(a)
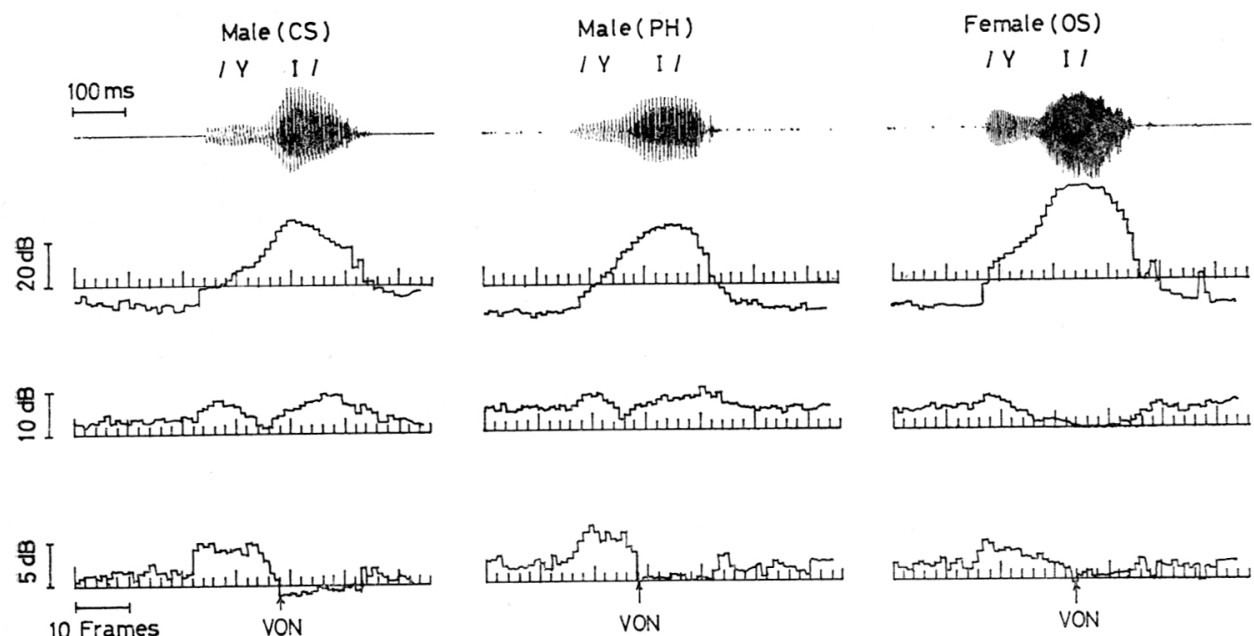

$v_{y}(2)$

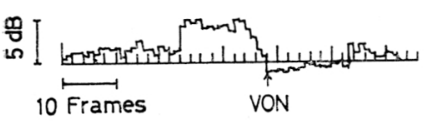

(b)

Fig. 4 Waveforms together with time variations of $v_{\nu}(0), v_{\nu}(1)$, and $v_{\nu}(2)$, of utterances of $/ \mathrm{yu} /$ spoken by two male and one female talkers in (a); and those of utterances of /yI/ spoken by the three talkers in (b). 


\subsection{Cepstral Distance Measure}

To measure the similarity of vocal tract characteristics between two frames ( $\nu$ and $\nu^{\prime}$ ), we use the following cepstral distance measure ${ }^{7}$ :

$$
\left[d_{\nu, \nu}\right]^{2}=\left[v_{\nu}(0)-v_{\nu^{\prime}}(0)\right]^{2}+\frac{1}{2} \sum_{m=1}^{M}\left[v_{\nu}(m)-v_{\nu^{\prime}}(m)\right]^{2} .
$$

The term $d_{\nu, \nu^{\prime}}$ can be interpreted as the $\mathrm{rms}$ distance between the log spectral envelopes. In the automatic segmentation procedure, the steady state as well as dynamic periods within a source word are detected by means of cepstral distance measures.

\subsection{Intensity of Log Spectral Envelope Com-} ponents in a Frequency Region

To estimate the intensity of log spectral envelope components in a $f_{1}-f_{2}$ region, we use the following equation $^{1)}$

$$
\begin{aligned}
B_{\nu}\left(k_{1}, k_{2}\right) & =\frac{\sum_{k=0}^{N-1} V_{\nu}(k) W(k)}{\sum_{k=0}^{N-1} W(k)} \\
& =v_{\nu}(0)+\frac{1}{2} \sum_{m=1}^{M} \frac{v_{\nu}(m) w(m)}{w(0)},
\end{aligned}
$$

where $k_{1}=N f_{1} / F$ ( $F$ is sampling rate), $k_{2}=N f_{2} / F$, $W(k)$ is the spectral window specifying the frequency region, and $w(m)$ is the inverse Fourier transform of $W(k)$. The following spectral window is used:

$$
W(k)=W(N-k)= \begin{cases}1, & k_{1} \leq k \leq k_{2} \\ 0, & \text { otherwise } .\end{cases}
$$

In the procedure, the voiced-unvoiced decision and the evaluation of the intensity of the log spectral envelope components in the first formant frequency region are performed by means of $B_{\nu}\left(k_{1}, k_{2}\right)$. For the former, $k_{1}$ is $2(\simeq 80 \mathrm{~Hz})$ and $k_{2}$ is $8(\simeq 320 \mathrm{~Hz})$. For the later, $k_{1}$ is $5(\simeq 200 \mathrm{~Hz})$ and $k_{2}$ is $23(\simeq 900$ $\mathrm{Hz})$.

\section{SEGMENTATION PROCEDURE}

The segmentation procedure is primarily implemented to establish the DS boundary locations within a spoken utterance. Since those locations have reference to some locations, e.g., vowel onset, vowel offset, one of the transitional frames, etc., determining such reference location(s) first is an important tactic of establishing the DS boundary locations. The routines of determining the same location, however, may differ from source word to source word. Generally, to find one of the transitional frames, we determine a vowel frame first and then search toward the transitional region for that by means of a prominent cue (mostly a cepstral distance measure for a $\mathrm{CV}$ source word, and the cepstral gain for a VC source word). The vowel onset as well as offset, which, based on the rules for defining the demisyllables in chapter 2 , is the most important reference location for establishing the vowel-side boundary location, is generally determined by searching from the transitional frame toward the vowel by means of a cepstral distance measure. The vowel-side boundary location is generally established based on a rule as to where the vowel cut would be made. The determination of consonant-side boundary location involves prominent cues particular to each type of consonants.

However, the order of determining the locations are, as the case may be, sometimes different from the order shown above. We shall describe the segmentation procedure in details by grouping the source words into 4 broad categories: (1) all $\mathrm{CV}$ source words except those beginning with the semivowels $/ 1 /, / w /$, and $/ y /$; (2) CV source words beginning with the semivowels shown above; (3) source words of vowels; and (4) all VC source words.

When we smooth frame-to-frame variation of a segmental cue (e.g., in the course of picking a minimum or maximum value), we use a 3 -point window with coefficients $1 / 4,1 / 2,1 / 4$; hereinafter, $\tilde{x}$ will denote the smoothed $x$.

\subsection{Category 1}

Figure 5 (a) shows the flow diagram for extracting DSs from the CV source words of this category: the $\mathrm{CV}$ source words beginning with the stops, the affricates, the trill $/ r /$, the nasals, and the fricatives. The steps and routines of obtaining the DS boundary locations are as follows:

\subsubsection{Find one of the vowel frames (VOW)}

For each following vowel, the intensity of the log spectral envelope components in the first formant frequency region (about $200 \sim 900 \mathrm{~Hz}$ ) computed by Eq. (4), $B_{2}(5,23)$, are stable and larger than those of initial consonants. The frame with maximum $\tilde{B_{\nu}}(5,23)$ is usually within the steady state period of the following vowel; this frame is thus considered as the VOW (if two or more frames are 
found, the latest frame in time is chosen).

4.1.2 Find one of the $\mathrm{CV}$ transitional frames (CVTR)

Since the following vowel has a steady state period, cepstral distances computed for all pairs of vowel frames tend to be small. Hence for the initial stops, nasals, and the trill $/ \mathrm{r} /$, search from the VOW toward the consonant for the nearest frame whose cepstral distance from the VOW, $d_{\nu, \text { vow, }}$ exceeds or equals a given threshold $\left(\theta_{1}\right)$. We have found that $d_{\nu, \text { vow }}$ was normally below $5 \mathrm{~dB}$; yet the distances between two distinct frames ranged from $12 \mathrm{~dB}$. The threshold $\theta_{1}$ can then be any value in the range of $5 \sim 12 \mathrm{~dB}$; here we use $8 \mathrm{~dB}$.

For the initial fricatives and affricates, the determination of CVTR is as follows: Search from the VOW toward the initial consonant for the frame whose $\tilde{v}_{\nu}(1)$ is minimum and that is nearest to the VOW if two or more frames are found, consider that as one of the frames within the consonant region (call it $\mathrm{CON}$ ); then search in the CON-VOW region for the frame whose $v_{\nu}(1)$ performs maximum upward change from its preceding frame $\left[\left\{v_{\nu}(1)-\right.\right.$ $\left.v_{\nu-1}(1)\right\}$. is maximum], and that is nearest to the CON if two or more frames are found.

For the phoneme $/ \mathrm{h} /$, the vocal tract position for the vowel which follows $/ \mathrm{h} /$ is assumed during the production of $/ \mathrm{h} /$. The most prominent contrast between the production of $/ \mathrm{h} /$ and the production of the vowel that follows $/ h /$ is the excitation: the phoneme $/ \mathrm{h} /$ is produced by exciting the vocal tract by a steady air flow, i.e., without the vocal cords vibrating, whereas the vowel that follows $/ \mathrm{h} /$ is produced by exciting the vocal tract by a periodic impulse train. A voiced-unvoiced decision cue is thus the most prominent cue signaling the transitional region. A previous study ${ }^{1)}$ has suggested that the intensity of the log spectral envelope components in the fundamental frequency region, $B_{2}(2,8)$, has a very good performance in making voiced-unvoiced decision, i.e., once a voiced-unvoiced threshold has been determined for one talker, it can similarly well be applied to a new talker. In our determining the voiced-unvoiced threshold to be used throughout the procedure, two $B_{\nu}(2,8)$ histograms, one of which was of all the voiced sounds and the other was of all the unvoiced sounds of the spoken source words of the talker CS, were displayed on the same axes; the value at the intersection of the two histograms
$(-24 \mathrm{~dB})$ was chosen. We have noted that the chosen value is the same as that previously chosen based on such histograms for various talkers.

The determination of CVTR is to search from the VOW toward the consonant for the nearest frame whose $B_{\nu}(2,8)$ is below the threshold $\left(\theta_{2}\right)$; the immediately following frame is considered as the CVTR.

\subsubsection{Find the vowel onset (VON)}

Since it was found that cepstral distances for pairs of vowel frames were below $5 \mathrm{~dB}$, the determination of VON is to search from the CVTR toward the VOW for the nearest frame whose distance from its preceding frame, $d_{\nu, \nu-1}$, is below $5 \mathrm{~dB}$ (threshold $\theta_{3}$ ). The reason for determining the CVTR by means of the threshold $\theta_{1}$ before determining the VON is to first estimate a range in which the VON is expected to be found, i.e., to assure that the VON is not going to be searched for in the region of the initial consonant, which sometimes also involves a steady state region. Normally, the VON is found next to the CVTR.

\subsubsection{Establish the final segmental boundary (FSB)}

Since each following vowel has static characteristics, we can make a vowel cut anywhere within the steady state period. Assuming that the duration of every following vowel is longer than $80 \mathrm{~ms}$ (each source word should be uttered with a long vowel), we choose the seventh frame behind the VON as the FSB for all CV demisyllables (see rules 1, 2, and 3 for defining the demisyllables in chapter 2) in order to unify their vowel durations.

\subsubsection{Find the initial segmental boundary (ISB)}

For the initial consonants involving dynamic characteristics, which include the stops, $/ \mathrm{dzh} /, / \mathrm{t} / /$, and the trill $/ \mathrm{r} /$, the ISB must be the first frame of the initial consonant (see rule 3 for defining the demisyllables). For all the voiced stops, the voiced affricate $/ \mathrm{dzh} /$, and the $/ \mathrm{r} /$ (which is voiced), the ISB is determined by means of the $B_{\Downarrow}(2,8)$, the voicedunvoiced decision cue, with the threshold $\theta_{2}$. For the unvoiced stops and the unvoiced affricate $/ \mathrm{t} / /$, the earliest frame involving explosion is considered as the ISB. That frame is determined by means of $v_{\nu}(0)$, the cepstral gain, which suddenly rises at the explosion by about 10 $25 \mathrm{~dB}$ from the level of silence (about $-40 \mathrm{~dB}$ ). The explosion can thus be detected by a given cepstral gain threshold $\left(\theta_{4}\right)$. The threshold $\theta_{4}$ was chosen to be $-35 \mathrm{~dB}$.

For each initial nasal, which has rather static 

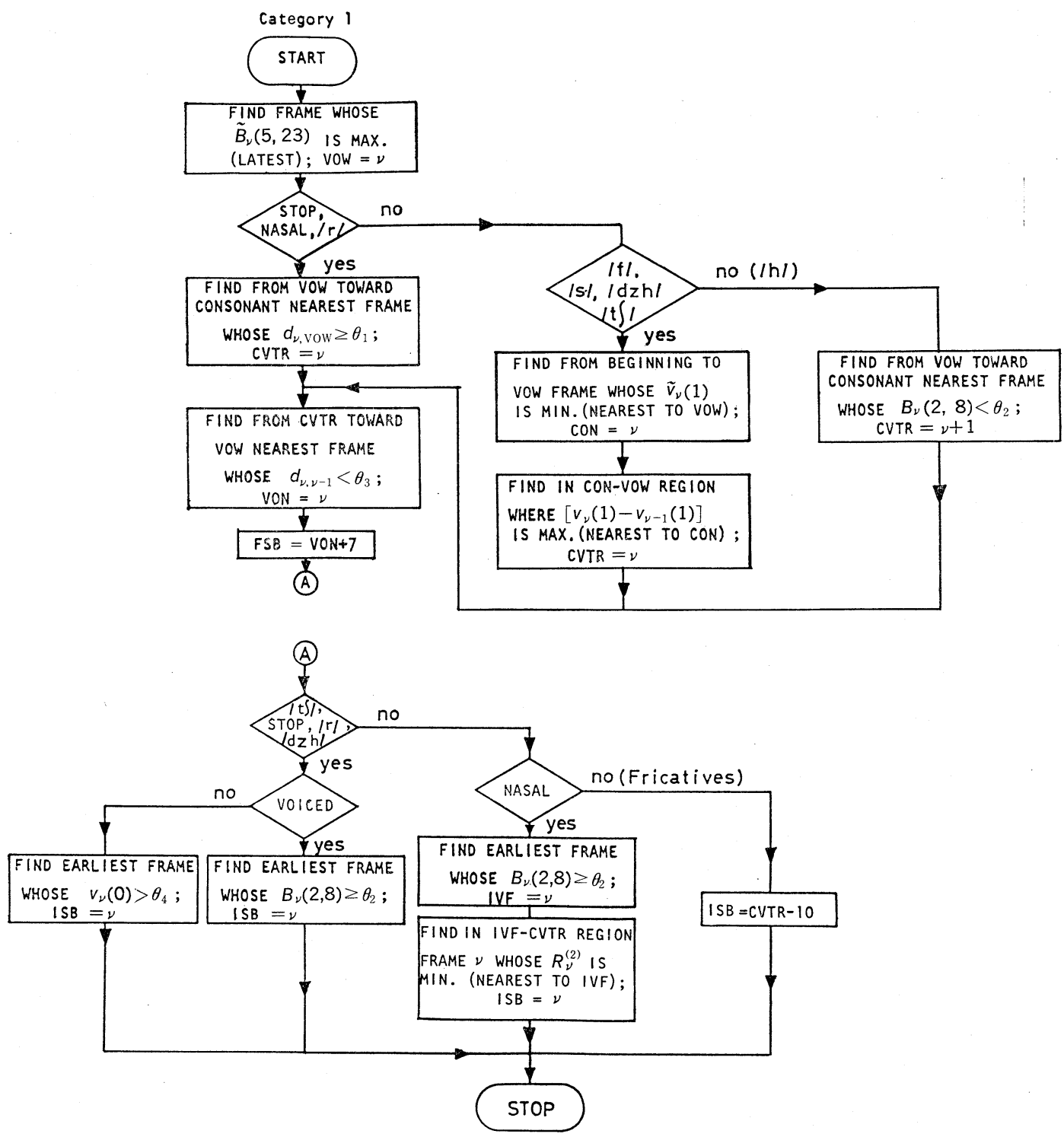

Fig. 5 (a) Flow diagrams for extracting demisyllables from source words of category 1.

characteristics, the ISB should be a frame in the most steady state region because it will be used as an interpolating boundary, one of the frames between which interpolation is performed. The determination of ISB is as follows: Search from the first silence frame for the earliest frame whose $B_{2}(2,8)$ exceeds or equals the threshold $\theta_{2}$; consider that frame the initial voiced frame (IVF). Then, search in the IVF-CVTR region for the frame that shows the smallest value of the average distances from the two immediately following frames [the minimum of the set $\left(d_{\nu, \nu+1}+d_{\nu, \nu+2}\right) / 2, \quad$ IVF $\leq \nu \leq \mathrm{CVTR} ; R_{\nu}{ }^{(n)}$ will be used of the term $\left(d_{\nu, \nu+1}+d_{\nu, \nu+2}+\cdots+\right.$ $\left.d_{\nu, \nu+n}\right) / n$ hereinafter], and that is nearest to the IVF if two or more frames are found (see rule 1 for defining the demisyllables).

The fricatives $/ \mathrm{s} /, / \mathrm{f} /$, and $/ \mathrm{h} /$ are unvoiced continuant sounds, each of which has a steady state period. Unlike in the case of the initial nasals, the ISB will not be used as an interpolating boundary 


\section{SARAVARI and S. IMAI: AUTOMATIC EXTRACTING DEMISYLLABLES}

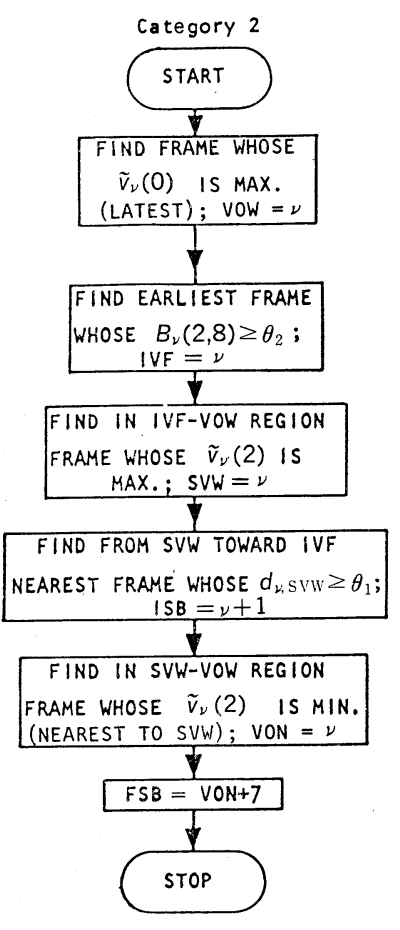

(b)
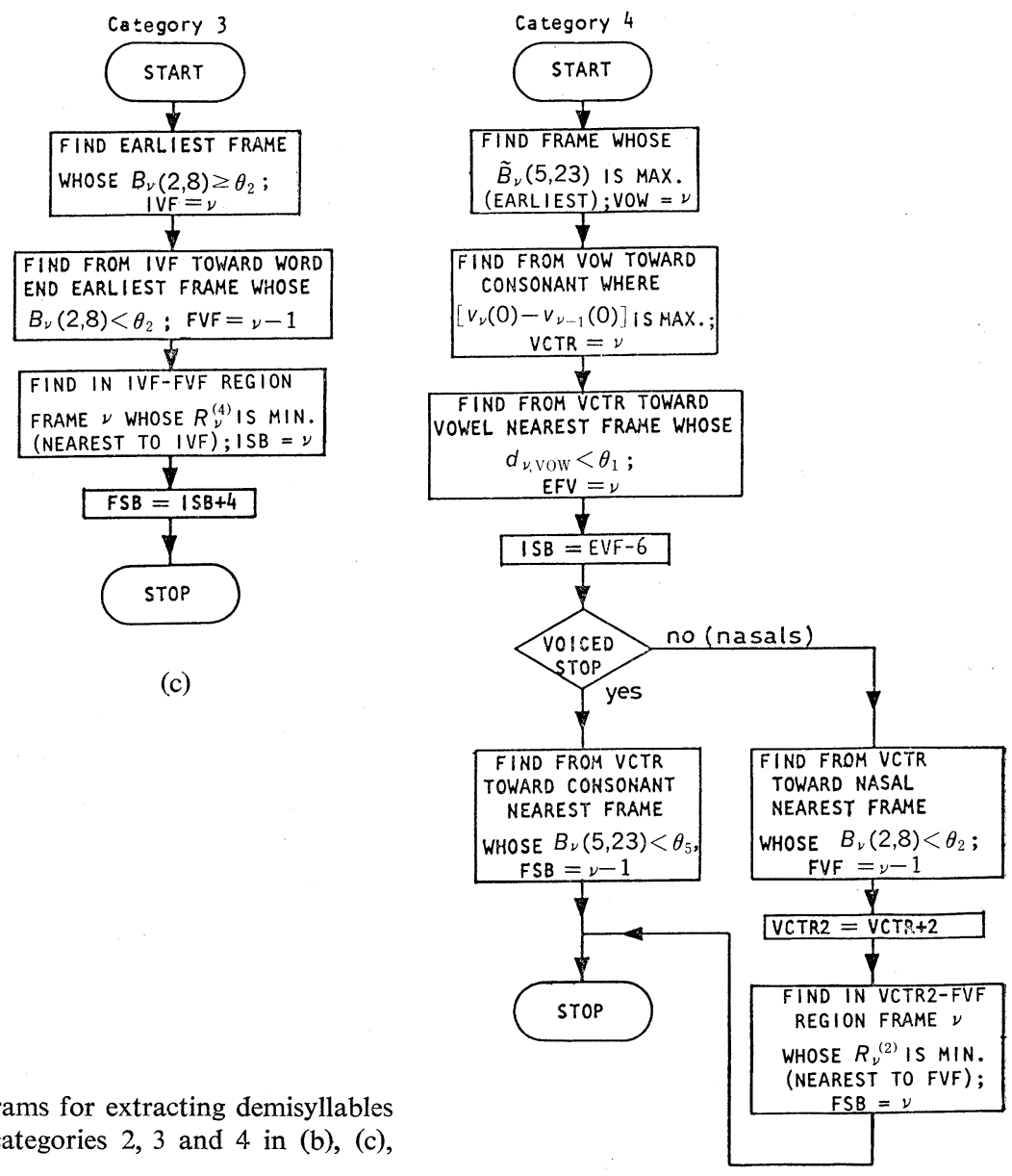

(d) because it is unvoiced. Hence, we may choose any frame in the steady state region to be the ISB. Normally, a fricative has a long steady state period, and the transitional region between it and the following vowel is short (1-2 frames). Besides, as described in section 3.2, the determination of the CVTR is very accurate. Accordingly, we use a rule as to where the fricative should be cut (here $100 \mathrm{~ms}$ from the CVTR, see rule 2 for defining the demisyllables) for establishing the ISB; hence, the tenth frame in front of the CVTR is chosen as the ISB.

\subsection{Category 2.}

Figure 5 (b) shows the flow diagram for extracting DSs from the CV source words of this category: the CV source words beginning with the semivowels $/ 1 /, / w /$, and $/ y /$.

\subsubsection{Find one of the vowel frames (VOW)}

During each of the semivowels, the cepstral gain, $v_{\nu}(0)$, is normally lower than that of a vowel that follows it (see Fig. 4) because the signal power during the initial semivowel is weaker than that during the following vowel. The cepstral gain usually becomes maximum somewhere just behind the vowel onset. Hence, the frame with maximum $\tilde{v}_{\nu}(0)$ is searched for and considered as the VOW; if two or more frames are found, the latest frame in time is chosen.

4.2.2 Find the initial segmental boundary (ISB)

Since each of the semivowels is a rather static sound, any frame in the steady state region may be chosen as the ISB; here, the earliest steady state frame is chosen (see rule 1 for defining the demisyllables) in order to keep the initial semivowel as long 
as possible (the determination of ISB for the initial semivowels differs from that for the initial nasals because the semivowels are normally not as long as the nasals; determining ISB by means of the $R_{\nu}^{(2)}$ may make the semivowels so short that they will not be of good quality). The ISB is established as follows: Find the initial voiced frame (IVF) by means of the $B_{2}(2,8)$ with the threshold $\theta_{2}$. Search in the IVF-VOW region for the frame whose $\tilde{v}_{\nu}(2)$ is maximum, and that is nearest to the IVF if two or more frames are found; call that frame SVW (one of the initial semivowel frames [see section 3.3]). Search from the SVW toward the IVF for the nearest frame whose $d_{\nu, \mathrm{Svw}}$ exceeds or equals the threshold $\theta_{1}$; then establish the following frame in time as the ISB.

4.2.3 Find the vowel onset (VON)

Search in the SVW-VOW region for the frame whose $\tilde{v}_{\nu}(2)$ is minimum, and that is nearest to the SVW if two or more frames are found (see section 3.3).

4.2.4 Establish the final segmental boundary (FSB)

The FSB is the seventh frame behind the VON (see section 4.1.4).

\subsection{Category 3}

Figure 5 (c) shows the flow diagram for extracting DSs from the source words of this category: the vowels. Each vowel has a steady state period. The segmentation is done so that each DS contains five most steady state frames (see rule 6 for defining the demisyllables) as follows:

(1) Find the initial voiced frame (IVF) by means of the $B_{\nu}(2,8)$ with the threshold $\theta_{2}$.

(2) Search from the IVF toward the end of the word for the final voiced frame (FVF) also by means of the $B_{\nu}(2,8)$ with the threshold $\theta_{2}$.

(3) Search in the IVF-FVF region for frame $v$ that shows minimum $R_{\nu}{ }_{\nu}^{(4)}\left[\left(d_{\nu, \nu+1}+d_{\nu, \nu+2}+\cdots+\right.\right.$ $\left.d_{\nu, \nu+4}\right) / 4$ ], and that is nearest to the IVF if two or more frames are found; establish the first and last frames of that set ISB and FSB, respectively.

\subsection{Category 4}

The source words of this category are words of the VC form. Figure 5 (d) shows the flow diagram for extracting DSs from the source words of this category.

4.4.1 Find one of the vowel frames (VOW)

The maximum of $\tilde{B}_{\nu}(5,23)$ is searched for. If two or more frames are found, the earliest frame in time is chosen.

4.4.2 Find one of the VC transitional frames (VCTR)

Each of the final consonants is begun by an abrupt closure somewhere in the vocal tract, which makes the values of $v_{\nu}(0)$ much smaller than those during the vowel that precedes it. Thus, the determination of VCTR is to search from the VOW toward the end of the word for the frame whose $v_{\nu}(0)$ drops most rapidly from its preceding frame [i.e., $\left\{v_{2}(0)-\right.$ $\left.v_{\nu-1}(0)\right\}$ is maximum], and that is nearest to the VOW if two or more frames are found.

4.4.3 Find the vowel offset, or ending frame of preceding vowel (EFV)

Search from the VCTR toward the vowel for the nearest frame whose $d_{\nu \text {, vow }}$ is below the threshold $\theta_{1}$.

4.4.4 Establish the initial segmental boundary (ISB)

The ISB is the sixth frame in front of the EFV (see rules 4 and 5 for defining the demisyllables). 4.4.5 Find the final segmental boundary (FSB)

For each final stop, the vocal cords stop vibrating and the sound disappears soon after the closure is complete; the duration of the stop is quite short. During the transitional region and the final stop, the first and second formants are clearly seen whereas the higher formants obscured. The parameter specifying the intensity of the log spectral envelope components in the first formant frequency region $\left[B_{\nu}(5,23)\right]$ appears to be a prominent cue in determining the end of each final stop; the $B_{\nu}(5,23)$ usually drops rapidly from the position of the ending frame of each final stop to that of silence. Thus, the determination of FSB for each final stop is to search from the VCTR toward the end of the word for the earliest frame whose $B_{\nu}(5,23)$ is below a given threshold $\left(\theta_{5}\right)$; the preceding frame is considered as the FSB (see rule 5 for defining the demisyllables). The threshold $\theta_{5}$ is chosen to be $-45 \mathrm{~dB}$, which is slightly above the $B_{2}(5,23)$ values for silence.

For each final nasal, the vocal cords continue vibrating for a while after the closure is complete; the pressure is released through the nose. The final nasals share the characteristics of steady state resonance with the corresponding initial nasals. Hence, like in the case of the initial nasals, the FSB should be one of the most steady state frames. 
The determination of FSB for each final nasal involves the voiced-unvoiced decision cue and cepstral distance measures as follows: search for the final voiced frame (FVF) by means of the $B_{2}(2,8)$ with the threshold $\theta_{2}$, call the second frame behind the VCTR VCTR2, then search in the VCTR2-FVF region for the frame with minimum $R_{\nu}{ }^{(2)}$, and that is nearest to the FVF if two or more frames are found (see rule 4 for defining the demisyllables).

\section{PERFORMANCE OF THE PROCEDURE}

The automatic procedure was used to create DS inventories for two new arbitrary Thai talkers: one male and one female. The recording was made in a quiet room using a high quality microphone and recorder. To advise each talker as to how to pronounce each source word, we first played the recorded source word spoken by the talker CS. After a slight pause, the talker repeated the word. Once the talker uttered a source word, a native observer evaluated it, and, replaying the "standard" source word, asked the talker to repeat the word whenever he did not agree upon (each phoneme within the source word must be clearly heard, and undesirable sounds such as sounds of lips, breath, etc., must be kept as small as possible).

To check the accuracy of the automatic procedure, we used a trained native linguist to carefully establish the DS boundary locations within each source word based on looking at the waveform, log spectral envelopes, and the segmental cues, the rules for defining the DS described in chapter 2, and listening to synthetic sounds of the extracted DS and of the source word produced from the extracted DS (the synthesis rules can be found in Ref. 2)). The order of manual determining the locations was the same as that of automatic one. The trained linguist assured that no better synthetic sound was obtained upon changing any of the DS boundary locations. Accordingly, we shall assume that the manually obtained set of DSs is most suitable for use in a speech synthesis system (we have also found that Thai speech with good quality can be produced from such demisyllables ${ }^{2,3}$ ).

Comparing the automatically obtained DS boundary locations with the corresponding manually obtained locations, we found that the automatically obtained locations were within three frames from the manually obtained locations for both talkers.
For the male talker, $94.2 \%$ of the automatically obtained locations coincided with the corresponding manually obtained locations, and $3.5 \%, 1.9 \%$, and $0.4 \%$ differed by one, two, and three frames, respectively; for the female talker, $95.7 \%$ coincided, and $3.3 \%, 0.8 \%$, and $0.2 \%$ differed by one, two, and three frames, respectively. The above results are comparable with those of using the automatic procedure to extract the DSs from the spoken source words of the talker CS: $95.5 \%$ coincided, and $2.3 \%$ and $0.2 \%$ differed by one and two frames, respectively.

Table 1 shows the differences between the manually and automatically obtained locations. For each talker, the 1-frame, 2-frame, and 3-frame differences of both the ISB and FSB are shown for every category of source words and for every type of consonants for $\mathrm{CV}$ and $\mathrm{VC}$ source words. Since the vowel cut was made at the point of a certain distance from the VON (in case of $\mathrm{CV}$ ) or from the EFV (in case of VC), then the FSB differences and the ISB differences correspond to the VON differences (in case of $\mathrm{CV}$ ) and the EFV differences (in case of VC), respectively. The other intermediate locations need not to be concerned, since the errors of establishing those locations will consequently give rise to ISB and/or FSB errors. The total numbers of boundary location differences versus the total number of boundary locations (ISB + FSB) are in the last column of the part for each talker. The element(s) in the bottom of each column is (are) the sum(s) of other elements in that column. No difference occurred for the vowels (all the source words of category 3 ) and the CV source words beginning with the fricatives (category 1) for both talkers. No difference occurred for the $\mathrm{CV}$ source words beginning with the unvoiced stops (category 1) for the male talker; only one marginal 1-frame difference was found for the female talker. For some CV source words beginning with voiced stops (category 1), there was a tendency to cut off some of the buzz region which may exists in some instance before the explosion because the effect of voicing of the buzz was sometimes not so clear. However, to our ears, the errors were not enough to damage the intelligibility of the initial voiced stops. For some CV source words beginning with semivowels (category 2), the vowel onset (VON) location error sometimes occurred when the transition was rather smooth. That error usually gave rise to 


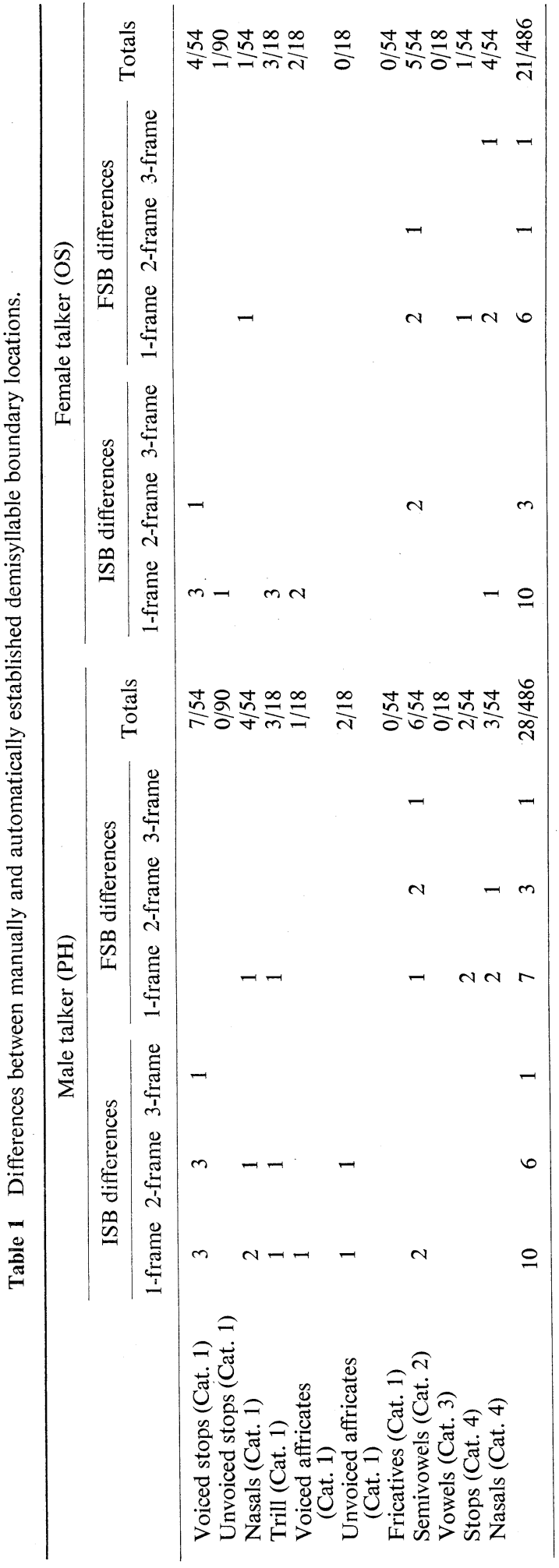

error in establishing the vowel-side boundary locations (usually making the vowel of the DS longer than that it should be, which, however, was not detrimental in any way to the intelligibility of the vowel). For the other source words, the differences that occurred tended to be 1-frame and might be regarded as marginal differences. In addition, it was found that no frame of silence was contained in the DSs, and that the ISB and FSB differences were not both found in a DS. It can also be seen from the table that the automatic procedure performed similarly well for both new talkers, i.e., same patterns of DS boundary differences.

The facts that most of the automatically obtained locations were the intended locations, and that the few differences that occurred tended to be within two frames suggest that synthetic speech with a good quality can also be obtained from each of the automatically obtained sets of DSs, and that the automatic procedure co-operated with few manual corrections can fast provide the best set of DSs for each talker. To obtain that set, perhaps the best way is to display the waveforms, log spectral envelopes, segmental cues, etc., of the source words and adjust, if necessary, the automatically obtained locations until all criteria for defining the DSs are satisfied. Even by that method, establishing each DS boundary location with the automatically obtained location as a basis seems to be much easier and less time-consuming than establishing it all manually.

\section{DISCUSSION AND SUMMARY}

This paper has described in details a simple technique for automatically extracting demisyllables from Thai source words of the forms $\mathrm{CV}, \mathrm{VC}$, and $\mathrm{V}$. The procedure contains segmentation routines that are particular to each category of source words and to each type of consonants within the words of the forms $\mathrm{CV}$ and VC. The segmental cues are all derivable from the cepstrum representing a log spectral envelope that approximately passes through peaks of the fine structure. All the segmental cues, routines, and thresholds have been determined based on the spoken source words of one male talker.

The automatic procedure was found to be very accurate in establishing DS boundary locations for two new arbitrary talkers: one male and one female. Comparisons indicated that the automatically obtained DS boundary locations were within three frames ( 1 frame is $10 \mathrm{~ms}$ ) from the corresponding 


\section{SARAVARI and S. IMAI: AUTOMATIC EXTRACTING DEMISYLLABLES}

intended locations. For the male talker, $94.2 \%$ of the automatically obtained locations were the intended locations; and $3.5 \%, 1.9 \%$, and $0.4 \%$ differed from the intended locations by one, two, and three frames, respectively; for the female talker, $95.7 \%$ were the intended locations; and $3.3 \%, 0.8 \%$, and $0.2 \%$ differed from the intended locations by one, two, and three frames, respectively. The above results are comparable with the results of using the automatic procedure to extract the DSs from the spoken source words of the old talker: $97.5 \%$ were the intended locations, and $2.3 \%$ and $0.2 \%$ differed from the intended locations by one and two frames, respectively.

Most of the routines involve minimum or maximum value picking; based on the results, those routine tended to work similarly well for both talkers. The five thresholds $\left(\theta_{1} \sim \theta_{5}\right)$ tended to be unambiguous since they were normally between two distinct ranges of values. The thresholds $\theta_{1}$ and $\theta_{3}$ are both used to distinguish whether a distance is that between the two frames with different vocal tract characteristics or it is that between those with the same characteristics; these two distances were always found to be considerably different. The voicedunvoiced threshold $\theta_{2}$ chosen based on the data of the old talker (CS) is found to be the same as that previously chosen based on data of various talkers for use in a speech analysis-synthesis system. Thus, it can be thought that this threshold can similarly well be applied to most talkers. The cepstral gain threshold $\theta_{4}$ and the threshold $\theta_{5}$, which involves in evaluating the intensity of the log spectral envelope components in the first formant frequency region $\left[B_{\nu}(5,23)\right]$, are used to distinguish whether a frame is silence or that involving initial unvoiced plosive and whether a frame is silence or that involving final voiced plosive, respectively. The range of cepstral gains for the initial unvoiced plosives and the range of cepstral gains for silence as well as the range of
$B_{\nu}(5,23)$ values for the final voiced plosives and the range of $B_{\nu}(5,23)$ values for silence are most likely to be-and they really were-considerably different. Based on the performance test, it was found that the cues, routines, and thresholds could similarly well be applied to both new talkers.

We conclude that the present automatic procedure for extracting demisyllables from isolated source words promises a very good first estimate set of demisyllables, and that co-operated with few manual corrections, provides a set of demisyllables suitable for use in speech synthesis-by-rule systems. The automatic procedure seems to be most helpful, especially to an amateur, for the course of creating a suitable set of demisyllables for each of the talkers for whom computer voice is required, which can reduce the tediousness and save much time.

\section{REFERENCES}

1) Y. Abe and S. Imai, "Speech synthesis from CVsyllable cepstral parameters," Trans. IECE J64-D, 861-868 (1981) (in Japanese).

2) C. Saravari and S. Imai, "A demisyllable approach to speech synthesis of Thai-A tone language," J. Acoust. Soc. Jpn. (E) 4, 97-106 (1983).

3) C. Saravari and S. Imai, "Synthesis of Thai speech from demisyllable cepstral parameters," Acoust. Lett. 6, 32-35 (1982).

4) L. R. Rabiner, A. E. Rosenberg, J. G. Wilpon, and T. M. Zampini, "A bootstrapping training technique for obtaining demisyllable reference patterns," J. Acoust. Soc. Am. 71, 1588-1595 (1982).

5) S. Imai and Y. Abe, "Spectral envelope extraction by improved cepstral method," Electr. Commun. Jpn. 62-A, 10-17 (1979).

6) C. Saravari and S. Imai, "A segmentation method in speech synthesis of Thai," Acoust. Lett. 6, 11-14 (1982).

7) T. Kitamura and S. Imai, "Spectral distortion and quality of synthesized speech in cepstral speech analysis-synthesis system," Trans. IECE J65-A, 478-484 (1982) (in Japanese). 